\title{
The Use of Topical Compounded Analgesic Creams in Neuropathic Pain by the Primary Care Physician
}

\author{
Jan M Keppel Hesselink ${ }^{1^{*}}$ and David J Kopsky² \\ ${ }^{1}$ Institute for Neuropathic Pain, Spoorlaan 2a, 3735 MV, Bosch en Duin, Netherlands
}

${ }^{2}$ Institute for Neuropathic Pain, Vespuccistraat 64-III, 1056 SN, Amsterdam, Netherlands

*Corresponding author: Jan M Keppel Hesselink, Institute for Neuropathic Pain, Spoorlaan 2a, 3735 MV, Bosch En Duin, The Netherlands, Tel: 31628671847 ; E-mail: jan@neuropathie.nu

Received date: December 21, 2017; Accepted date: December 27, 2017; Published date: December 30, 2017

Copyright: (C) 2017 Hesselink JMK, et al. This is an open-access article distributed under the terms of the Creative Commons Attribution License, which permits unrestricted use, distribution, and reproduction in any medium, provided the original author and source are credited.

\begin{abstract}
The primary care physician often has to help diabetic patients suffering from localized neuropathic pain. Most of the current anti-epileptics and anti-depressants however, especially when prescribed to the elderly patients, have troublesome drawbacks: drug-interactions, side effects and tolerability issues. Topical compounded creams might be a good alternative, especially since it is easy to test in the general practice to discover responders to these creams.

At our Institute for Neuropathic Pain we see many patients suffering from peripheral neuropathic pain. We have developed a number of compounded creams based on amongst others amitriptyline $10 \%$, ketamine $10 \%$, clonidine $0.2 \%$, baclofen $5 \%$, and phenytoin $10 \%$. Many patients profit from such topical formulations and we will describe how a primary care physician can fast decide whether as specific compounded cream is useful for a patient. Patients suffering from peripheral neuropathic pain, as in diabetic neuropathy, chronic idiopathic axonal polyneuropathy (CIAP) and chemotherapy induced polyneuropathy (CIPN) as well as from complex regional pain syndrome (CRPS), seem often to be responsive to such topical approach. Meanwhile, more than 800 Dutch medical doctors have been prescribing one or more of our compounded creams, and patients and medical doctors from abroad frequently contact us to explore the therapeutic options for their patients based on these creams. We will give some practical tips how to differentiate between responders and non-responders.
\end{abstract}

Keywords: Topiceuticals; Cream; Phenytoin; Amitriptyline; Ketamine; Baclofen

\section{Introduction}

At our Institute for Neuropathic Pain we see mostly neuropathic pain patients, already often seen by the primary care physician. Many are suffering from peripheral symmetrical painful neuropathies, such as in diabetes (as chronic sensorimotor symmetrical polyneuropathy), chemotherapy induced polyneuropathy (CIPN) and chronic idiopathic axonal neuropathy (CIAP). Diabetes mellitus (DM) is a growing health problem with epidemic proportions. Most of the patients suffering from neuropathic pain in the daily practice of the primary care physician are such DM patients. Primary care physicians play an increasing role in the treatment of DM patients, and for instance in the United States treat at least $90 \%$ of the 24 million patients with DM [1].

Many of the anti-neuropathic pain drugs however, such amitriptyline, duloxetine, pregabalin and gabapentin have serious drawbacks in this elderly population due to drug-interactions side effects and tolerability issues. Alternatives therefore are welcome [2]. Topical treatment has been documented to have an excellent risk/ benefit ratio, safety and tolerability profile and continued efficacy throughout long-term treatment [3]. Treatments such as plasters or patches containing lidocaine or capsaicin however are difficult in handling and individualized therapy with such topical formulations is complicated. This is one of the reasons why topical compounded creams are gaining more acceptance in the general practice.
There are many active pharmaceutical ingredients possible in such creams, for instance amitriptyline, clonidine, ketamine, baclofen and phenytoin. In our practice, we gained experience during 9 years prescribing such compounding creams, and we summarized our experience in a number of articles [4-6]. In the period 2008-2011 we started compounding a number of creams based on amitriptyline and ketamine and found out that most of the approaches at that time selected a dose too low, based on only $1 \%$ to $5 \%$. We found that such analgesics needed to be compounded in creams containing at least $5 \%$ and preferably $10 \%$ of the active ingredient [7]. The same holds true for phenytoin, where we also prefer a $10 \%$ compounded cream [8].

We gathered most experiences prescribing the following concentrations in compounded creams: amitriptyline 10\%, ketamine $10 \%$, baclofen $5 \%$, clonidine $0.2 \%$ and phenytoin $10 \%$. Due to small drawbacks, such as some local burning of amitriptyline, the abuse potential of ketamine, the potential antihypertensive effects of clonidine and the putative muscle relaxing effects of baclofen, we currently prefer to start prescribing phenytoin cream $10 \%$. In certain cases, we have increased the dose to phenytoin $30 \%$ to create full response. Other compounds, such as gabapentin, are in our view not rational to add in compounded creams, as there are to date no targets identified in the skin. Its mechanism of action is only based on reaching sufficient plasma-levels of gabapentin in order to influence the receptors in the central nervous system. 


\section{A Practical Response Test to Identify Responders to a Topical Cream}

In case of a chronic distal symmetrical polyneuropathy such as in DM and CIPN, usually both feet are painful and burning with same intensity. This enables the physician to directly differentiate between responders and non-responders.

Using the simple 11-point numerical rating scale (NRS) for pain which 0 is no pain and 10 is the worst pain ever - we can let the patient score the pain in the left and the right foot independently. As most patients have symmetrical pain (e.g. pain in both feet), with comparable intensity, in 2013 we came on the idea to develop a simple on-site response test, were patients can compare the effect of an intervention on one foot to the other. We started by using the simplest design, an open response test, applying certain compounded analgesic creams on one foot and compare its effect to the untreated foot.

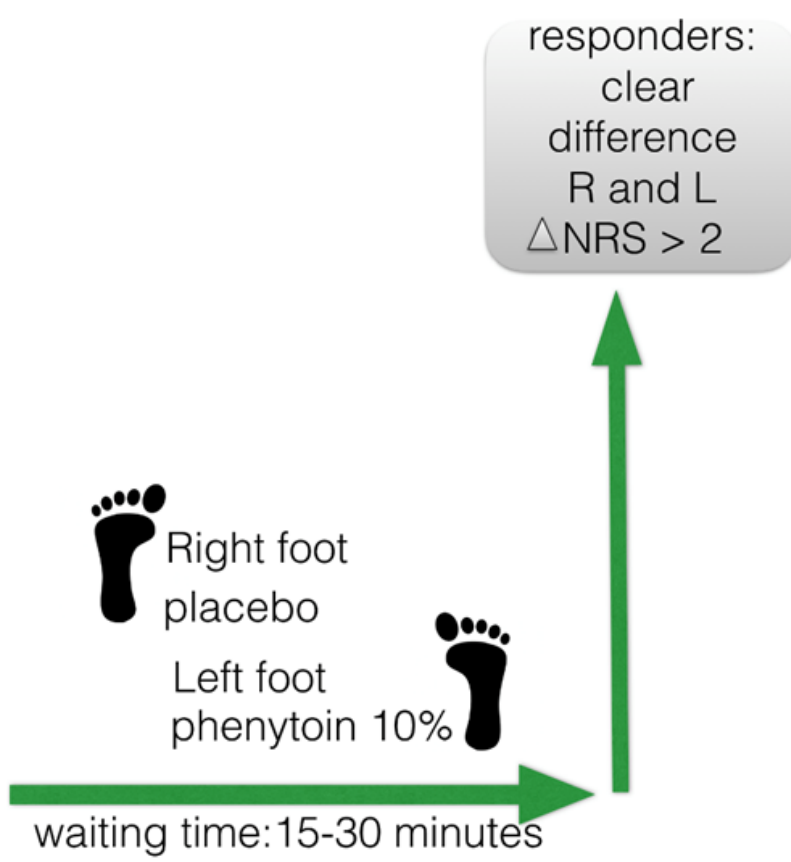

Figure 1: simple single-blind response test to evaluate for instance phenytoin $10 \%$ cream as the active treatment versus placebo, applying equal amounts of creams (1 fingertip unit) to both feet. After 15 to 30 minutes, responders feel a clear difference in pain intensity between both feet. If the difference between placebo and active cream is 2 or more, we prescribe the active cream.

At that time we noticed already that high dose topicals, such as phenytoin $10 \%$ to $20 \%$ cream, amitriptyline $10 \%$ cream and baclofen $5 \%$ often resulted in a fast response within minutes, and leading to a decrease of 2 points on the NRS or even more in responders. This led us to define responders as those patients who responded within 30 minutes with a decrease of at least 2-point reduction of pain as scored on the NRS. After application of an active cream on one foot, the patient subsequently sits in the waiting room, mostly reading a bit, until we ask the patient to come to our desk after 20 to 30 minutes.
Those patients which indicated a decrease of pain of at least 2 points on the NRS were prescribed subsequently the tested topical formulation. Most of these patients remained a responder, with a pain decrease of at least $50 \%$ or more during chronic use. Many of our patients have prescriptions of topical compounded creams valid for a year, and often ask renewals of such prescriptions. For instance, one of our patients treated with phenytoin $10 \%$ cream has now been treated successfully already for more than 3 years. In order to further objectify our test, we added a placebo cream to the response test, and applied placebo on the other foot, thus starting our single-blind placebocontrolled test paradigm (Figure 1).

We subsequently adapted our definition of a responder based on our gained experience: after 15 to 30 minutes, patients need to notice at least a difference of 2 points on the NRS in pain reduction between the active cream and to the placebo cream. Meanwhile, we are able to analyse cohorts of patients based on this single-blind response test and find our creams to be statistically superior compared to placebo. We will publish these findings soon, based on the single-blind response test comparing placebo to phenytoin cream $10 \%$.

\section{Summary and 'Hands-on'}

If a patient presents him/herself in the primary care practice, the patients first point of entry into the health care system, and is suffering from neuropathic pain in both feet, the physician can start asking the intensity of the pain, using the simple NRS. If a patient indicates the pain is present at both feet (in most cases) and has an intensity between 4 to 10 on the NRS a compounded cream containing an analgesic might be indicated. Especially elderly with comorbidities and polypharmacy might profit from topical analgesics. Compounded pain creams are nearly always safe to use, and interactions, adverse events and tolerability issues are extremely rare. The physician can ask the compounding pharmacist in the neighbourhood to give him some samples (e.g. amitriptyline $10 \%$ cream) and comparable tubes containing placebo, marking these as $\mathrm{A}$ and $\mathrm{B}$. He subsequently lets the patient administer cream A (one fingertip unit) on one foot and cream $\mathrm{B}$ on the other. The patient can wait in the waiting room for 15 to 45 minutes. The effect is mostly seen within 20 minutes. When 2 points or more difference in pain on the NRS is present between both feet supporting the active cream, subsequently the physician can prescribe the patient the active cream. This in the Netherlands also helps to get reimbursement from the health insurance, as we could make plausible in a single-blind design the efficacy of the cream. Based on our experience we feel that first-line use of topical agents may be of particular benefit in elder patients if safety and tolerability of oral therapies may be a concern [9].

\section{Conflict of Interest}

The authors are patent holders of two patents related to the topical formulations of phenytoin in the treatment of pain: Topical phenytoin for the use in the treatment of peripheral neuropathic pain and Topical pharmaceutical composition containing phenytoin and a (co-) analgesic for the treatment of chronic pain.

\section{References}

1. Davidson JA (2010) The increasing role of primary care physicians in caring for patients with type 2 diabetes mellitus. Mayo Clin Proc 85: S3-4. 
Citation: Hesselink JMK, Kopsky DJ (2017) The Use of Topical Compounded Analgesic Creams in Neuropathic Pain by the Primary Care Physician. J Gen Pract (Los Angel) 5: 345. doi:10.4172/2329-9126.1000345

Page 3 of 3

2. Derry S, Wiffen PJ, Kalso EA, Bell RF, Aldington D, et al. (2017) Topica analgesics for acute and chronic pain in adults - an overview of Cochrane Reviews. Cochrane Database Syst Rev 12: CD008609.

3. Pickering G, Martin E, Tiberghien F, Delorme C, Mick G (2017) Localized neuropathic pain: An expert consensus on local treatments. Drug Des Devel Ther 11: 2709-2718.

4. Keppel Hesselink JM, Kopsky DJ (2017) Topical compounded analgesic treatment in neuropathic pain: 8 years of experience. J Pain Manage Med 3: 128.

5. Kopsky DJ, Keppel Hesselink JM (2017) Topical phenytoin for the treatment of neuropathic pain. J Pain Res 10: 469-473.

6. Keppel Hesselink JM, Kopsky DJ (2017) Topical phenytoin cream in small fiber neuropathic pain: fast onset of perceptible pain relief. Int J Pain Relief 1: 15-19.
7. Kopsky DJ, Keppel Hesselink JM (2012) High doses of topical amitriptyline in neuropathic pain: two cases and literature review. Pain Pract 12: 148-53.

8. Kopsky DJ, Keppel Hesselink JM (2017) Phenytoin in topical formulations augments pain reduction of other topically applied analgesics in the treatment of trigeminal neuralgia. J Clin Anesth 38: 154-155.

9. Sommer C, Cruccu G (2017) Topical treatment of peripheral neuropathic pain: applying the evidence. J Pain Symptom Manage 53: 614-629. 